\title{
State determination in continuous measurement
}

\author{
A. C. Doherty, S. M. Tan, A. S. Parkins, D. F. Walls \\ Department of Physics, University of Auckland, Private Bag 92019, Auckland, New Zealand
}

(March 9, 1999)

\begin{abstract}
The possibility of determining the state of a quantum system after a continuous measurement of position is discussed in the framework of quantum trajectory theory. Initial lack of knowledge of the system and external noises are accounted for by considering the evolution of conditioned density matrices under a stochastic master equation. It is shown that after a finite time the state of the system is a pure state and can be inferred from the measurement record alone. The relation to emerging possibilities for the continuous experimental observation of single quanta, as for example in cavity quantum electrodynamics, is discussed.

42.50.Lc,03.65.Bz,42.50.Ct
\end{abstract}

\section{INTRODUCTION}

There is currently great interest in experiments which obtain useful information about a quantum system or state in single runs of an experiment. Very recently it has been possible to distinguish the quantum mechanical and classical models of the interaction of an atom with a mode of a high finesse optical cavity as the result of continuous monitoring of the output light while a single atom passes through the cavity [1]. As a result of this continuous monitoring the quantum mechanical backaction of the measurement process may be expected to have a significant effect on the evolution of individual runs of the experiment. Moreover, it may be possible in the future to modify the evolution of the system through feedback based on this continuous measurement [2]. Current experimental technology, such as that described in [1], is reaching the point at which determining the state of the system and observing the effects of backaction and feedback in a single run of an experiment is a real possibility. With this situation in mind, we discuss the identification of the state of the system following a period of continuous observation and the extent to which this state can be tracked, taking into account factors such as imperfect initial knowledge of the state and imperfect detection efficiency. We focus on a model of continuous position measurement of a mechanical oscillator which is relevant to the experiment of Mabuchi et al. but which also has relevance to other instances of interferometric position monitoring such as gravitational wave detection.

The problem of describing quantum systems undergoing continuous measurement has attracted much theoretical interest in recent years. As discussed by Wiseman [3] these theories admit a variety of interpretations; as tools for efficient stochastic calculation of ensemble averages in lieu of solving master equations [4], as equations describing the evolution of systems conditioned on measurements [5] -7] and as a description of the evolution of a system coupled to an environment, in which collapse of the wavefunction is supposed to be associated with the coupling to the environment [8]. Here, we take the second viewpoint namely that the conditioned state represents the observer's best description of the system state given the results of the continuous measurement process. Adopting the first or third viewpoints one is led to describe the system by a pure state vector throughout the evolution although the reasons for doing so are somewhat different in each case. By contrast, a description of one's conditioned state of knowledge necessarily requires mixed states in order to account for incomplete knowledge of the system. From this viewpoint the fundamental equation for the conditioned evolution is the stochastic master equation (SME) [9]. This is able to account for the effects of mixed initial states, imperfect detection efficiencies and the existence of unmeasured couplings to the environment. However, to date, relatively little work has attempted to address the evolution of the conditioned state in any of these situations [10]. In this paper we consider a system which is simple enough that almost all the work can be done analytically and which admits a treatment of all of these imperfections. This helps in developing intuition about the role of SME's and their possible relevance to experiments.

A projective measurement has the property that if the result of the measurement is known, the state after the measurement is pure, and depends only on the measurement result. It would be hoped that in a continuous measurement there would be some finite interval of time after which the measurement has effectively given rise to a projection, so that the system is placed in a particular state which depends only on the sequence of measurement results and which can be calculated without knowledge of the initial state. If the resulting state is pure then a stochastic Schrödinger equation (SSE) would be a perfectly adequate tool for describing the subsequent system evolution. In this paper we investigate the conditions which lead to such an effective collapse and over what timescale it takes place. This is made possible by considering density matrices and the SME rather tha wave vectors and the SSE. In a real experiment there will also be uncontrolled, unmeasured couplings of the system to the environment and in this case the effects of the measurement will compete not only with the coherent internal dynamics of the system but also with the 
randomizing effects of the coupling to the bath. This may lead to mixed conditioned states even after long periods of continuous measurement and limit the observer's ability to make inferences about the system state. Understanding the process by which the conditioned state may collapse onto a pure state and the effects of noise as described by the SME allows us to define conditions under which continuous measurements in real experiments are approximations to ideal measurements.

This paper is organized as follows. In Sec. II we establish our simplified model of the continuous position measurement of an oscillator and solve the SME for Gaussian initial states. We find the time over which the secondorder moments approach their steady-state values and calculate the entropy of the conditioned state as it becomes pure. In Sec. III we discuss the classical problem of state identification for the noisy measurement of the the position of an oscillator and derive a kind of uncertainty principle relating the observation and process noises if the classical model is to reproduce the SME. In Sec. IV we show that the time-scale over which the second-order moments of the conditioned state reach their steady state is the same as that over which the conditioned state is completely determined by the measurement record. Section $\square$ discusses the effect of heating, noise and detection inefficiency on these conclusions. Finally in Sec. VI we summarize and make some comments about future extensions of this work.

\section{SOLVING THE STOCHASTIC MASTER EQUATION}

\section{A. A Generalized Model for Continous Position Measurement}

In this paper we shall consider the abstract model of continuous position measurement discussed by Caves and Milburn [11.9] with a harmonically bound rather than a free measured particle. Projective position measurements are imagined to be made on a sequence of meters coupled briefly to the system and the limit of very frequent meter interactions and very broad initial meter position distributions is taken. This leads to a continuous evolution for the system of interest. Although this model should correspond in some limit to any continuous position measurement of a single oscillator at the standard quantum limit, one system which does realize it at least approximately is the dispersive regime of single atom cavity quantum electrodynamics (QED). In this system the position of an atom inside a high-finesse optical cavity causes a phase shift of the field driving the cavity which can be monitored by homodyne detection 12 15. The SME for the conditioned evolution of the system in its Itô form is [16,9] $d \rho_{\mathrm{c}}=-i\left[H_{\mathrm{sys}}, \rho_{\mathrm{c}}\right] d t+2 \alpha \mathcal{D}[x] \rho_{\mathrm{c}} d t+\sqrt{2 \alpha} \mathcal{H}\left[e^{-i \phi} x\right] \rho_{\mathrm{c}} d W$

The superoperators $\mathcal{D}[c]$ and $\mathcal{H}[c]$ acting on a density matrix $\rho$ are $\mathcal{D}[c] \rho=c \rho c^{\dagger}-\frac{1}{2} c^{\dagger} c \rho-\frac{1}{2} \rho c^{\dagger} c$ and $\mathcal{H}[c] \rho=$ $c \rho+\rho c^{\dagger}-\operatorname{Tr}\left(c \rho+\rho c^{\dagger}\right) \rho$ where $c$ is an arbitary operator. We will imagine that the atom is harmonically bound,

$$
H_{\mathrm{sys}}=\frac{p^{2}}{2 m}+\frac{m \omega^{2} x^{2}}{2} .
$$

The constant $\alpha$ describes the strength of the measurement interaction and in the cavity QED example depends on the strength of the coherent driving and of the damping of the cavity. For the moment we will consider a onesided cavity and perfect detection so that all the output light is detected. This assumption will be relaxed in Sec. (V). In a generalization of the Caves and Milburn model we will allow projective measurements of any quadrature of the meters, not just position, since this can be realized in the cavity QED experiments by varying the phase $\phi$ of the local oscillator in the homodyne detection of the output light. The resulting measurement current $i=\frac{d Q}{d t}$ (suitably scaled) is

$$
d Q=\cos (\phi)\langle x\rangle_{\mathrm{c}} d t+\sqrt{\frac{1}{8 \alpha}} d W .
$$

This stochastic master equation with the full dependence on $\phi$ was discussed by Diosi [17] in the context of a phenomenological model of position measurement through photon scattering where the kind of measurement made on the scattered photon determines the value of $\phi$. Clearly if we choose $\phi=0, \pi$ the homodyne detection is an effective measurement of the atomic position, whereas if $\phi=\pi / 2,3 \pi / 2$ the measurement results are independent of the system state and only contain information about the noisy potential seen by the atom. For $\phi=\pi / 2,3 \pi / 2$ the conditioned evolution is linear in the system state [9,15]. This is somewhat like a continuous quantum eraser where the continuous measurement of one quadrature of the probe system destroys the position information written into the other quadrature of the probe. For $\phi=0$ the SSE corresponding to this model in the case of pure states of the system has been considered in 18,19. In this work we will use a simpler means of solving the evolution equations which straightforwardly applies to mixed states.

It has been shown by Jacobs and Knight [19] that the SSE corresponding to Eq. (2.1) is one for which Gaussian pure states 20 remain Gaussian and pure under the evolution. Thus, if the system is initially in a mixture of Gaussian pure states, the conditioned state will remain Gaussian under the SME (2.1). This property holds true for single mode systems where the Hamiltonian is at most quadratic in $x$ and $p$ and the operator $c$ appearing in the Lindblad term $\mathcal{D}[c]$ is linear in $x$ and $p$ and in all likelihood for multimode linear systems also. 
If we restrict ourselves to Gaussian initial states - for example to thermal states of the oscillator - then there are only five quantities which completely define the state $-\langle x\rangle,\langle p\rangle,\left\langle(\Delta x)^{2}\right\rangle=\left\langle x^{2}\right\rangle-\langle x\rangle^{2},\left\langle(\Delta p)^{2}\right\rangle=\left\langle p^{2}\right\rangle-\langle p\rangle^{2}$ and $\langle\Delta x \Delta p\rangle_{\text {sym }}=\frac{1}{2}(\langle x p+p x\rangle-2\langle x\rangle\langle p\rangle)$. From now on we will use $\langle c\rangle$ to indicate the conditioned expectation value $\operatorname{Tr}\left(c \rho_{\mathrm{c}}\right)$. The requirement that the initial states be Gaussian is not unduly restrictive since these are typically the states that are most stable in linear systems and would therefore be a reasonable assumption for an initial state. Moreover, there is numerical evidence that non-Gaussian pure initial states become approximately Gaussian under the stochastic Schrödinger equation evolution corresponding to the SME (2.1) on timescales fast compared to those considered here [21,22]. It has also been shown that in at least one such linear system an arbitary density matrix can eventually be written as a probabilistic sum over Gaussian pure states after sufficient evolution subject to the unconditioned master equation 23. In considering such a linear system we are in effect specifying a semiclassical evolution, since the equations of motion for the Wigner function of the state in the unconditioned evolution are similar to the classical Liouville equations for a phase space density, see for example [24]. However we here include the important quantum feature of the measurement backaction, which is represented by the fact that the momentum diffusion is determined by the accuracy with which the particle's position is monitored [9]. So, as the noise in the position measurement is decreased by increasing $\alpha$, the momentum diffusion expressed by the Lindblad term in the SME (2.1) increases.

Following Breslin and Milburn [25] we can derive a system of differential equations for the first and second-order moments from the SME (2.1) since $d\langle c\rangle=\operatorname{Tr}\left(c d \rho_{\mathrm{c}}\right)$. A similar calculation is performed in [23]. We define the dimensionless quantities $\tilde{x}=$ $\langle x\rangle / \sqrt{\hbar / 2 m \omega}, \tilde{p}=\langle p\rangle / \sqrt{\hbar m \omega / 2}$, the second-order moments $V_{x x}=2 m \omega\left\langle(\Delta x)^{2}\right\rangle / \hbar, \quad V_{p p}=2\left\langle(\Delta p)^{2}\right\rangle / \hbar m \omega$, $V_{x p}=2\langle\Delta x \Delta p\rangle_{\text {sym }} / \hbar$ and a dimensionless parameter describing the relative strengths of the measurement and harmonic dynamics, $r=m \omega^{2} / 2 \hbar \alpha$. The Heisenberg uncertainty principle now requires that $V_{x x} V_{p p} \geq 1$. A pure state has the property that $V_{x x} V_{p p}-V_{x p}^{2}=1$, representing the fact that a Gaussian pure state is a minimum uncertaintly state for some pair of conjugate quadrature variables 20]. In terms of these new quantities, the Itô stochastic differential equations for the first and secondorder moments and the measured photocurrent are

$$
\begin{aligned}
d \tilde{x} & =\omega \tilde{p} d t+\sqrt{\frac{2 \omega}{r}} \cos (\phi) V_{x x} d W \\
d \tilde{p} & =-\omega \tilde{x} d t+\sqrt{\frac{2 \omega}{r}}\left(\cos (\phi) V_{x p}-\sin (\phi)\right) d W \\
d \tilde{Q} & =\cos (\phi) \tilde{x} d t+\sqrt{\frac{r}{2 \omega}} d W \\
\frac{1}{\omega} \frac{d V_{x x}}{d t} & =2 V_{x p}-\frac{2}{r} \cos ^{2}(\phi) V_{x x}^{2}
\end{aligned}
$$

$$
\begin{aligned}
\frac{1}{\omega} \frac{d V_{p p}}{d t}= & -2\left(1-\frac{\sin (2 \phi)}{r}\right) V_{x p}+\frac{2}{r} \cos ^{2}(\phi) \\
& -\frac{2}{r} \cos ^{2}(\phi) V_{x p}^{2}, \\
\frac{1}{\omega} \frac{d V_{x p}}{d t}= & V_{p p}-\left(1-\frac{\sin (2 \phi)}{r}\right) V_{x x} \\
& -\frac{2}{r} \cos ^{2}(\phi) V_{x x} V_{x p} .
\end{aligned}
$$

As in [25], the Itô rules for stochastic differential equations [26] and the properties of Gaussian states 27] result in deterministic equations for the conditioned secondorder moments which are decoupled from the equations for the means. The constant term in the equation for $V_{p p}$ refers to the momentum diffusion due to the position measurement and remains in the master equation for the unconditioned evolution. The non-linear terms describe the conditioning of the state on the measurement. The noisy contribution to the equation for $d \tilde{x}$ seems a little like a stochastic impulsive force, however it is perhaps better to think of this term as updating the expected position given the measurement result $d \tilde{Q}$ in analogy with classical Bayesian state estimation.

Equations like those above for the second-order moments of the conditioned state arise very frequently in classical continuous-time observation and control problems. They can be collected into a Riccati matrix differential equation [28] for the covariance matrix,

$$
\begin{aligned}
\frac{d}{d t} V & =\omega(C-V B V-D V-V A) \\
V & =\left(\begin{array}{ll}
V_{x x} & V_{x p} \\
V_{x p} & V_{p p}
\end{array}\right) \\
A & =D^{T}=\left(\begin{array}{l}
0 \\
-\left(1-\frac{\sin (2 \phi)}{r}\right)
\end{array}\right) \\
B & =\left(\begin{array}{ll}
\frac{2 \cos \phi}{r} & 0 \\
0 & 0
\end{array}\right) \\
C & =\left(\begin{array}{ll}
0 & 0 \\
0 & \frac{2 \cos \phi}{r}
\end{array}\right)
\end{aligned}
$$

A single variable Riccati equation which arose from the stochastic Schrödinger equation for this system was found and solved in [18].

In practice it may not be homodyne but rather heterodyne detection which can be experimentally achieved with noise at the quantum limit [1]. In this case, the local oscillator is detuned from the cavity frequency by a frequency $\Delta_{\text {het }}$ which is large compared to all system frequencies, with the result that the phase $\phi$ changes very rapidly. The quantum theory of heterodyne detection is described by Wiseman and Milburn [29]. The appropriate conditioned evolution can be described by averaging all the trigonometric functions of $\phi$ in the evolution equations (2.4) except where they are multiplied by Itô increments. Thus the equations 
for the second order moments are exactly those for homodyne detection with $\phi=0$ where $r$ is replaced by $2 r$, corresponding to halving the signal to noise ratio of the measurement. Considering the stochastic integrals $\int_{t}^{t+\delta t} \cos \left(\Delta_{\text {het }} t^{\prime}\right) d W\left(t^{\prime}\right), \int_{t}^{t+\delta t} \sin \left(\Delta_{\text {het }} t^{\prime}\right) d W\left(t^{\prime}\right)$, in the limit firstly of infinite $\Delta_{\text {het }}$ then of infinitesimal $\delta t$, leads to equations for the first order moments under heterodyne detection

$$
\begin{aligned}
& d \tilde{x}=\omega \tilde{p} d t+\sqrt{\frac{\omega}{r}} V_{x x} d W_{1} \\
& d \tilde{p}=-\omega \tilde{x} d t+\sqrt{\frac{\omega}{r}}\left(V_{x p} d W_{1}-d W_{2}\right)
\end{aligned}
$$

where $d W_{1}$ and $d W_{2}$ are independent Wiener increments. Again this is formally identical to the evolution with homodyne detection and $\phi=0$ where $r$ is replaced by $2 r$ and in which a second independent noise process $d W_{2}$ is present. Scaled versions of the two quadrature components of the experimental photocurrent are given by

$$
\begin{aligned}
& d \tilde{Q}_{1}=\tilde{x} d t+\sqrt{\frac{r}{\omega}} d W_{1}, \\
& d \tilde{Q}_{2}=d W_{2} .
\end{aligned}
$$

Again note the replacement of $r$ by $2 r$ in the equation for $d \tilde{Q}_{1}$ as compared to the equation for $d \tilde{Q}$ with $\phi=0$. So if the quadrature-phase $I_{2}(t)$ current is collected and used to account for the noisy potential, or alternatively fed back in order to compensate this evolution, then heterodyne detection is equivalent to homodyne detection with half the signal to noise ratio as far as the motional state is concerned.

\section{B. Steady State Conditioned Variances}

For all phases of the local oscillator $\phi \neq \pi / 2,3 \pi / 2$ the second-order moments possess a steady state. For example if $\phi=0$

$$
\begin{aligned}
V_{x x}^{\mathrm{ss}} & =\frac{1}{\sqrt{2}} r \sqrt{\sqrt{1+\frac{4}{r^{2}}}-1,} \\
V_{p p}^{\mathrm{ss}} & =\frac{1}{\sqrt{2}} r \sqrt{1+\frac{4}{r^{2}}} \sqrt{\sqrt{1+\frac{4}{r^{2}}}-1,} \\
V_{x p}^{\mathrm{ss}} & =\frac{1}{2} r\left(\sqrt{1+\frac{4}{r^{2}}}-1\right),
\end{aligned}
$$

which defines a pure state that agrees with the solution given in 18$]$. The steady states are found to have exactly the same second order moments regardless of the initial purity of the system. Assuming ideal detection, the observer is eventually able to ascribe a pure state to the system. When the harmonic oscillator dynamics dominates over the measurement $(r \gg 1)$ the steady conditioned state is approximately a coherent state with $V_{x x}^{\mathrm{ss}} \simeq V_{p p}^{\mathrm{ss}} \simeq 1, V_{x p}^{\mathrm{ss}} \simeq 1 / r$. If the measurement dynamics dominate $(r \ll 1)$ then $V_{x x}^{\mathrm{ss}} \simeq \sqrt{r}, V_{p p}^{\mathrm{ss}} \simeq \frac{2}{\sqrt{r}}, V_{x p}^{\mathrm{ss}} \simeq 1$ and the conditioned state is strongly squeezed in position, as one would expect for a position measurement which is rapid enough to overcome the internal dynamics of the system. The product of the position and momentum variances is greater than that required by the Heisenberg uncertainty principle as a result of the internal Hamiltonian which gives a non-zero correlation $V_{x p}$. Finally, the scaling we have chosen for the variances makes the limit of the free particle appear singular, however this limit exists and the results agree with Belavkin's [18]. Pure steady states also exist for other values of $\phi$ but the full expressions are rather complicated so we will just consider two special cases. If the oscillator dynamics dominate $(r \gg 1)$ the steady conditioned states are insensitive to the local oscillator phase leaving the conditioned state nearly in a coherent state $V_{x x}^{\mathrm{ss}} \simeq 1+\sin (2 \phi) / 2 r, V_{p p}^{\mathrm{ss}} \simeq$ $1-\sin (2 \phi) / 2 r, V_{x p}^{\mathrm{ss}} \simeq \cos ^{2} \phi / r$. If $r \ll 1$ the conditioned state is strongly dependent on the choice of local oscillator phase,

$$
\begin{aligned}
V_{x x}^{\mathrm{ss}} & \simeq \frac{\sqrt{r}}{|\cos \phi|} \sqrt{1 /|\cos \phi|+\tan \phi}, \\
V_{p p}^{\mathrm{ss}} & \simeq \frac{2}{\sqrt{r}} \sqrt{1 /|\cos \phi|+\tan \phi}, \\
V_{x p}^{\mathrm{ss}} & \simeq 1 /|\cos \phi|+\tan \phi .
\end{aligned}
$$

Rather surprisingly it is possible for some phases of the local oscillator that the momentum variance is in fact smaller than the position variance. This is because the measurement for non-trivial $\phi$ is a simultaneous measurement of the position of the oscillator and the momentum kicks to which it is being subjected and this can result in a more sharply defined momentum than position. This is really only a possibility for phases of the local oscillator where there is very little position information in the record.

In the cases $\phi=\pi / 2,3 \pi / 2$ the differential equations for the second order moments of the state are simply those that result from the unitary evolution of a simple harmonic oscillator and the SME (2.1) describes a stochastic unitary evolution. As a result there is no steady state for the moments, which grow according to the conventional Schrödinger equation. In this case the measurement current is white noise. In 21] it was noted that where a Lindblad operator is hermitian there exists an unravelling of the master equation which does not localize the conditioned state. The reason for this is clear in this context, such an unravelling corresponds to a measurement in which the observer obtains no information about the system state. 


\section{Timescale for Determination of a Pure State through Measurement}

The matrix Riccati equation (2.5a) has an analytic solution given by Reid [28]. Where $U(t)$ and $W(t)$ obey the linear coupled matrix equations

$$
\begin{aligned}
& \frac{d}{d t} U=A U+B W \\
& \frac{d}{d t} W=C U-D W
\end{aligned}
$$

and for times where $U(t)$ is non-singular, the solution for the covariance matrix is $V(t)=W(t) U^{-1}(t)$. The full solution for all the second-order moments and arbitary initial conditions is complicated and not particularly illuminating. In order to expose the general form of the solution we will just consider the position variance in the case of an initial state of the oscillator with $V_{x x}(0)=V_{p p}(0)=V_{0}, V_{x p}(0)=0$,

$$
\begin{gathered}
V_{0}\left(c^{2} \cosh 2 b t+b^{2} \cos 2 c t\right) \\
V_{x x}(t)=\frac{+\left(1+V_{0}^{2}\right)(c \sinh 2 b t-b \sin 2 c t)}{\left(b^{2}+c^{2}\right)+V_{0}\left(c^{3} \sinh 2 b t+b^{3} \sin 2 c t\right)} \\
+\left(1+V_{0}^{2}\right)\left(c^{2} \sinh ^{2} b t-b^{2} \sin ^{2} c t\right)
\end{gathered}
$$

We have scaled time by the harmonic oscillation frequency $\omega$. When $2 b t \gg 1$ the system is close to its steady state value regardless of $V_{0}$. The non-linearity of the terms describing the conditioning of the system state cause the time over which the conditioned state becomes pure to be independent of the initial temperature of the state. For definiteness we will define a collapse time $\tau_{\text {col }}=2 / b \omega$ as being the time at which the state has become effectively pure. When $r>1$ this collapse time is $\tau_{\text {col }} \simeq 2 r / \omega=m \omega / \hbar \alpha$ since in this regime $b \simeq 1 / r$. In this regime $c \simeq 1+1 / 2 r^{2}$ and there are many oscillations of the particle before the state is determined. That the time to determine a particular pure state of the system should increase with the frequency seems reasonable since unexpected values of the measurement current could be due to a mistaken idea of the position of the particle, the white noise in the measurement record or to motion due to the oscillation and these possibilities will be more difficult to distinguish if the atomic motion is fast. For smaller $r$, the measurement is becoming very good and this estimate for the collapse time is optimistic since it is hard to determine the state of the system in less than one period of the mechanical oscillation. Reductions in the conditioned momentum variance will only occur as the Hamiltonian evolution creates a correlation between the position and the momentum of the state. Even if the harmonic potential were absent a continuous measurement of position will give some information about the momentum of the particle. When $r \ll 1$ the particle is essentially free as far as the measurement is concerned and the time for the state reduction to occur turns out to be $\tau_{\text {col }} \simeq \sqrt{8 m / \hbar \alpha}$ which is determined solely by the strength of the measurement and the mass of the particle. In this situation $b \simeq c \simeq 1 / \sqrt{r}$. Increasing the measurement coupling means that the time for the measurement to take place is reduced and in the limit of infinite $\alpha$ the model essentially describes a projective measurement of the position. If heterodyne detection is used rather than homodyne detection then $r$ is replaced by $2 r$ in the above equations with obvious implications for the timescale of the system collapse.

A pure state describes a situation in which an observer has maximal information about the system. A mixed state describes less than maximal knowledge of system and it turns out that the amount of missing information, in an information theoretic sense, required to complete the specification of the state may be measured by the von Neuman entropy $S(\rho)=-\operatorname{Tr}(\rho \log \rho)$ of the density operator, see for example 30]. Thus the entropy allows us to quantify the extent to which the measurement has determined the state of the system at a given time and also the extent to which other environmental couplings limit what an experimenter can say about the system state. Another commonly used measure of the "mixedness" of a given density matrix is the linear entropy or purity $s(\rho)=1-\operatorname{Tr}\left(\rho^{2}\right)$. For a single mode Gaussian state these quantities are simple functions of the unitless "area" of the state in phase space $A=\sqrt{V_{x x} V_{p p}-V_{x p}^{2}}$ 31

$$
\begin{aligned}
& s(\rho)=1-\frac{1}{A}, \\
& S(\rho)=\frac{A+1}{2} \ln (A+1)-\frac{A-1}{2} \ln (A-1)-\ln 2 .
\end{aligned}
$$

Note that $A$ is just the determinant of the covariance matrix of the position and momentum probability distributions for the conditioned state. For a pure state $A=1, s(\rho) \rightarrow 0, S(\rho) \rightarrow 0$ and as the state becomes increasingly mixed it occupies a larger phase space area such that as $A \rightarrow \infty, s(\rho) \rightarrow 1, S(\rho) \rightarrow \infty$. As we would expect if the state is widely spread in phase space then our knowledge of the system is poor and the information needed to complete the description of the state is large.

The time evolution of the variances and the linear and von Neumann entropies of the conditioned state with $V_{x x}(0)=V_{p p}(0)=20, V_{x p}=0, \omega=1, r=20$ is plotted in Fig. (11). These parameters are chosen since the measurement dynamics are not fast enough to obscure the harmonic oscillation totally and because achieving very small values of $r$ will probably be difficult in practice. Several features of Fig. (1) are relevant. Firstly the initial very rapid reduction of the position variance is associated with the the first part of the measurement record making a reasonably accurate determination of the posi- 
tion. Then over the timescale of the harmonic oscillation the momentum variance also reduces as the dynamics correlate the position and momentum. Note that the reduction in momentum variance occurs only when there is a strong correlation between the position and the momentum. As $V_{x p}$ becomes small the position variance decays more rapidly and the reduction of the momentum variance slows. Eventually all the second-order moments decay to the steady state values predicted above. This initial fast reduction of the position variance is accompanied by a fast reduction of the von Neumann entropy which damps to zero as the system approaches steady state.

\section{Cavity QED Realization}

Although we have been considering an abstract model for continuous position measurement this work is motivated by emerging experimental possibilities in areas such as cavity quantum electrodynamics (QED). The position dependent phase shift induced by an atom strongly coupled to a high finesse optical cavity mode in the dispersive limit of cavity QED 12 realizes the abstract position measurement coupling considered here. It is currently possible to detect the presence of a single cold atom in the cavity through measurements of the output field [32,33. and great progress has been made in observing single atom events with broad bandwidth close to the dispersive regime [1]. The phase shift changes most quickly with position where the gradient of the field is greatest and if the atom is harmonically confined in this region then the model discussed above would be approximately realized. There is also the far off-resonant optical potential which will lead to large forces on the atom in this regime which would move the atom quickly away from this region of the standing wave. However an optical standing wave at a nearby frequency could in principle be tuned to cancel the ac-Stark shift of the ground state in the region of the harmonic confinement. In fact the dipole force could straightforwardly be included in our simplified model resulting in only minor changes for sufficiently strong harmonic confinement. For the moment we will not specify the exact source of the potential confining the atom, but the use of light forces from a far off-resonant optical standing wave or a standing wave in another mode of the cavity are possibilities. There is also experimental work aimed at confining ions in high finesse optical cavities which could realize such a system [34.

If we imagine a harmonic potential confining the atom to this region of the standing wave with some constant restoring force to overcome the dipole force then in the far-detuned and Lamb-Dicke limit the resulting SME would be exactly Eq. (2.1) above [15]. The constant $\alpha$ would then be equal to $2 g_{0}^{4} n k_{L}^{2} / \Delta^{2} \kappa$, where $g_{0}$ is the maximal single photon Rabi coupling in the cavity, $n$ is the number of photons present in the driven cavity in the steady state, $\Delta$ is the detuning between the atomic and cavity resonances - the external laser driving is on the cavity resonance, $\kappa$ is the cavity field decay rate and $k_{L}$ is the wave number for the light resonant inside the cavity. Using the parameters of [15] which are based on cavity parameters achieved by Hood et al. 33. gives $\alpha=2.4 \times 10^{20} \mathrm{~s}^{-1} \mathrm{~m}^{-2}$ and this determines the rate of decay of the off-diagonal terms in the position representation of the density matrix under the unconditioned evolution. This rather large number means that the density matrix elements $\left\langle x|\rho| x^{\prime}\right\rangle$ where $x$ and $x^{\prime}$ are separated by nine nanometers or around $1 \%$ of a wavelength will decay in the unconditioned evolution at the rate $2.0 \times 10^{4} \mathrm{~Hz}$. The decay of off-diagonal elements of the density matrix in a particular basis is often associated with decoherence and the emergence of classicality 35]. In this case the decoherence is due in to the measurement coupling. In [13] this decay of off-diagonal density matrix elements is described as state reduction, in this work we are interested in state reduction onto a pure state and the rate of this process is determined not only by $\alpha$ but also by the length scale of a typical pure state of the uncoupled system. Thus we found above that reduction onto a pure state took place in a time $\tau_{\text {col }} \simeq m \omega / 4 \hbar \alpha$ and the dependence on the length scale of the harmonic oscillator is clear. In order to find the rate of collapse onto a pure state in the conditioned evolution it is therefore also necessary to know the oscillation frequency of the atom due to its harmonic confinement. Assuming this is achieved optically, the value for $\omega / 2 \pi$ could be in the range of tens to hundreds of kilohertz. So for example in [15] the potential for a cesium atom resulting from the same cavity and driving parameters gives $\omega / 2 \pi=2 \sqrt{\hbar k_{L}^{2} / 2 m} \sqrt{g_{0}^{2} n / \Delta} / 2 \pi=180 \mathrm{kHz}$, while $\omega / 2 \pi \simeq 60 \mathrm{kHz}$ has been achieved for cesium in optical lattices by Haycock et al. [36. For such a hypothetical experiment with cesium we now have $r=5.6$ and $r=0.63$ respectively. Estimates, as outlined above, for the time for an experimenter to determine a pure state of the system through heterodyne detection are then $19 \mu$ s and $8.9 \mu \mathrm{s}$. Both these times are reasonably close to the minimum collapse time for this accuracy of detection which corresponds to the free particle limit with $\tau_{\text {col }} \simeq 3.9 \mu \mathrm{s}$. In current experiments detection efficiencies and bandwidths will have a significant effect on the information that can be gathered from the record. For trapped ions harmonic frequencies would be around an order of magnitude larger and state reduction times would then also be around an order of magnitude longer. However the cavity finesse used here for such experiments may be more difficult to achieve at typical frequencies of ion transitions and the size of the cavity will be limited by the ion trap electrodes. What is dramatic about this time is that it is so short, single cold atoms have been observed close to the centre of the cavity mode in the experiment at Caltech for times of the order of hundreds of microseconds. This is another confirmation of the extent to which this experiment operates near the Standard Quantum Limit 
of position measurement ( [37] and references therein) and an indication of the importance of continuous quantum measurement theory to its interpretation.

Note that it will be difficult to achieve very small values of $r$ for which the measurement dynamics dominate over the oscillation in the well. This is the result of the two main constraints on the applicability of the model. Firstly it is necessary that the harmonic potential confine the atom to well within a wavelength, thus justifying the Lamb-Dicke approximation in the master equation (2.1). this requires that the recoil frequency for the cavity transition $\omega_{\text {rec }}=\hbar k_{L}^{2} / 2 m$ be much smaller than the harmonic oscillation. Moreover attaining the dispersive regime requires that the saturation parameter $s=g_{0}^{2} n / \Delta^{2}$ be much smaller than one. It is possible to express $r$ in terms of these quantities: $r=\left(\omega / \omega_{\text {rec }}\right)(1 / 8 s)\left(\omega / \Gamma_{\text {cav }}\right)$ where $\Gamma_{\text {cav }}=g_{0}^{2} / \kappa$ is the cavity mediated spontaneous emission rate 38. Assuming that values of $\omega$ and $s$ are chosen to satisfy a particular level of approximation, the rate at which the conditioned states become pure is only increased by changing the cavity parameters through increasing $\Gamma_{\text {cav }}$ relative to the oscillation frequency. Moreover, since both the first two factors in this expression for $r$ must be large for the SME (2.1) to correspond to the cavity system, extremely large values of $\Gamma_{\text {cav }}$ are necessary to achieve rapid measurement of the system state.

\section{CLASSICAL ANALOGUE}

If we are to interpret the conditioned state as the best description of the observer's knowledge of the quantum mechanical state given the results of a series of measurements, we would expect a similarity between these equations and classical Bayesian state observation. The analogy between the SME (2.1) and Kalman filtering for a classical position measurement was discussed in [9] but only the equations for the position probability distributions were considered. Here we formulate the continuous time position measurement state observer for a classical harmonic oscillator and find that there is a close analogy between the SME and the classical theory for all moments of the conditioned probability distribution as long as we restrict ourselves to Gaussian states and allow for noisy driving of the classical oscillator.

The problem of noisy, classical, continuous time position measurement of a harmonic oscillator can be formulated

$$
\begin{aligned}
\frac{d x_{C}}{d t} & =\omega p_{C}, \\
\frac{d p_{C}}{d t} & =-\omega x_{C}+\sqrt{\frac{2 \omega}{s}} \epsilon, \\
\frac{d Q_{C}}{d t} & =a x_{C}+\sqrt{\frac{r}{2 \omega}} \eta, \\
E\left[\epsilon(t) \epsilon\left(t^{\prime}\right)\right] & =E\left[\eta(t) \eta\left(t^{\prime}\right)\right]=\delta\left(t-t^{\prime}\right), \\
E\left[\epsilon(t) \eta\left(t^{\prime}\right)\right] & =f \delta\left(t-t^{\prime}\right)
\end{aligned}
$$

We have used the same scaling of the variables as in the quantum problem. We imagine that as well as having an imperfect measurement of the system the oscillator is subject to a white noise force. There may be some correlation between the oscillator (plant or process) noise and the measurement noise and so $\epsilon$ and $\eta$ are correlated Wiener processes. As in the quantum mechanical case the limit of small $r$ is the limit of good position measurement.

The continuous time theory of Kalman filtering then provides the best estimate of the system state $\hat{x}, \hat{p}$ and the second-order moments of the posterior probability distribution $P\left(x_{C}, p_{C}\right)$ 39,

$$
\begin{aligned}
d \hat{x}_{C}= & \omega \hat{p}_{C} d t+a \sqrt{\frac{2 \omega}{r}} V_{x x} d W, \\
d \hat{p}_{C}= & -\omega \hat{x}_{C} d t+\left(a \sqrt{\frac{2 \omega}{r}} V_{x p}+f \sqrt{\frac{2 \omega}{s}}\right) d W, \\
\frac{1}{\omega} \frac{d V_{x x}}{d t}= & 2 V_{x p}-\frac{2}{r} a^{2} V_{x x}^{2} \\
\frac{1}{\omega} \frac{d V_{p p}}{d t}= & -2\left(1+\frac{2 a f}{\sqrt{r s}}\right) V_{x p}+\frac{2}{r} \\
& -\frac{2 f^{2}}{s}-\frac{2}{r} a^{2} V_{x p}^{2}, \\
\frac{1}{\omega} \frac{d V_{x p}}{d t}= & V_{p p}-\left(1+\frac{2 a f}{\sqrt{r s}}\right) V_{x x}-\frac{2}{r} a^{2} V_{x x} V_{x p} .
\end{aligned}
$$

Where $d W$ is an independent Wiener increment with $d W^{2}=d t$ proportional to the innovation process $d Q-a \hat{x}$. Note that the circumflex employed here indicates that the quantity is an estimate of the classical variable and not that it is a quantum operator. The moments of the posterior probability distribution have been given the same notation as the moments of the conditioned quantum mechanical state. If we make the identifications $s=r, a=\cos \phi, f=-\sin \phi$ then this system of equations is formally identical to the system which determines the evolution of the quantum mechanical conditioned state Eqns (2.4).

We see that there is a classical model of noisy position measurement for which the equations of motion for the posterior probability distribution of the classical state given by the Kalman filter reproduce the stochastic master equation. What is specifically quantum mechanical in the SME is that we cannot, even in principle, specify the process noise and the measurement noise separately. Classically one could imagine isolating the system sufficiently that $s$ is as large as we like. With $s \rightarrow \infty$ there would be no momentum disturbance on the atom and after a sufficiently long observation time the state of the system would be determined exactly so that $V_{i j}^{\mathrm{ss}}=0$. Clearly this does not correspond to a quantum state. However the quantum theory of the problem guarantees that any coupling to the system which gives position information about the state of the system must also disturb the momentum. This momentum dis- 
turbance must be sufficient that the conditioned state always obeys the Heisenberg uncertainty principle. Thus only some classically possible models of position measurement are allowed by quantum mechanics. For a given level of measurement noise, the process noise must be at least sufficient to ensure that the observer can never infer probability distributions for the position and momentum which do not constitute valid quantum states. This is a measurement-disturbance uncertainty relation [40] for continuous measurement; reducing the noise in the measurement must increase the noise in the evolution. This backaction noise does not however behave like classical process noise, its properties are entirely determined by the measurement. If we vary the basis of the measurement on the meter - vary $\phi$ - then we are able to alter the correlation between the measurement noise and the apparent process noise in the classical model.

We have found here that the symmetric moments of the conditioned state always obey a system of equations which also describes Kalman filtration for a classical problem, but that altering the specific quantum measurement alters both the observation and the process noise of the relevant classical problem. Since the symmetric moments are the moments of the Wigner function, for example [27], the conditioned Wigner function can be interpreted as the direct analogue of a classical posterior probability distribution for the system. This relationship is not going to be as straightforward for more complicated quantum systems where the conditioned Wigner function can be negative and cannot be interpreted as a probability distribution.

\section{DEPENDENCE ON INITIAL STATE}

We have established above that the conditioned state of the system can in priniciple become pure after a finite observation time. What might not be clear is that the state which results is uniquely determined by the measurement record. In an ideal projective measurement the probabilities of obtaining the various measurement results depend upon the initial state. However, once a result has been obtained the conditional state after the measurement depends on that result alone. In a similar way, for continuous measurements we find that, while the initial state affects the probability of obtaining particular measurement records, the conditional state following the measurement is determined by the particular measurement record which was obtained, provided that record sufficiently long. In particular if the initial state of the system is very poorly known to an observer then we might hope that there is effectively a maximum likelihood estimate of the system state which depends only on the measurement current and which converges to the actual system state within $\tau_{\text {col }}$. If propagating the stochastic master equation with the actual initial state of the system provides the a posteriori es- timate of the system state given the measurement results, then such a maximum likelihood estimate would result from propagating an initial state with very large position and momentum variances in the SME (2.1), say $V_{x x}=V_{p p}=V_{0} \rightarrow \infty, V_{x p}=0$, which gives a nearly flat prior probability distribution for the initial state. In this section we demonstrate that such a strategy does indeed work. Thus the purity of the conditioned density matrix indicates, as would be hoped, that there is only one pure state of the system at time $t>\tau_{\text {col }}$ which is consistent with the known sequence of measurement results.

Suppose that two observers, Alice and Bob say, postulate different initial states of the system $\rho^{\mathrm{A}}(0), \rho^{\mathrm{B}}(0)$, which we will continue to assume to be Gaussian. For example, Alice may have more information than Bob about the system in which case Bob would start with a more mixed initial density matrix reflecting his initial lack of knowledge. Given that they both recieve the same measurement record $I(t)$ and propagate their conditioned states according to the SME (2.1), it should be the case that at some time $t^{\prime}$, around $\tau_{\text {col }}$, Alice and Bob agree on the system state, so that $\rho^{\mathrm{A}}\left(t^{\prime}\right)=\rho^{\mathrm{B}}\left(t^{\prime}\right)$. From the previous section we know that after the time $\tau_{\text {col }}$ the second order moments of both conditioned states will be equal $V_{i j}^{\mathrm{A}}=V_{i j}^{\mathrm{B}}=V_{i j}^{\mathrm{ss}}$ and so we focus on the equations for the first order moments of the conditioned state for each observer

$$
\begin{aligned}
d x^{\mathrm{A}} & =\omega p^{\mathrm{A}} d t+\sqrt{\frac{2 \omega}{r}} V_{x x}^{\mathrm{A}} d W, \\
d p^{\mathrm{A}} & =-\omega x^{\mathrm{A}} d t+\sqrt{\frac{2 \omega}{r}} V_{x p}^{\mathrm{A}} d W, \\
d x^{\mathrm{B}} & =\omega p^{\mathrm{B}} d t+\sqrt{\frac{2 \omega}{r}} V_{x x}^{\mathrm{B}} d W^{\mathrm{B}}, \\
d p^{\mathrm{B}} & =-\omega x^{\mathrm{B}} d t+\sqrt{\frac{2 \omega}{r}} V_{x p}^{\mathrm{B}} d W^{\mathrm{B}}, \\
d Q & =x^{\mathrm{A}} d t+\sqrt{\frac{r}{2 \omega}} d W=x^{\mathrm{B}} d t+\sqrt{\frac{r}{2 \omega}} d W^{\mathrm{B}} .
\end{aligned}
$$

In this section we will omit the tildes used above to indicate that we have scaled the position and momentum to the natural units for the harmonic oscillator. The stochastic increment $d Q$ is the infinitesimal increment of the measured current which both Alice and Bob have access to. We can express the increment $d W^{\mathrm{B}}$ in terms of the other quantities $d W^{\mathrm{B}}=d W-\sqrt{2 \omega / r}\left(x^{\mathrm{B}}-x^{\mathrm{A}}\right) d t$, and find stochastic differential equations for the differences between the means $e_{x}=x^{\mathrm{B}}-x^{\mathrm{A}}, e_{p}=p^{\mathrm{B}}-p^{\mathrm{A}}$,

$$
\begin{aligned}
d e_{x}= & \omega e_{p} d t-\frac{2 \omega}{r} V_{x x}^{\mathrm{B}} e_{x} d t \\
& +\sqrt{\frac{2 \omega}{r}}\left(V_{x x}^{\mathrm{B}}-V_{x x}^{\mathrm{A}}\right) d W, \\
d e_{p}= & -\omega\left(1+\frac{2}{r} V_{x p}^{\mathrm{B}}\right) e_{x} d t
\end{aligned}
$$




$$
+\sqrt{\frac{2 \omega}{r}}\left(V_{x p}^{\mathrm{B}}-V_{x p}^{\mathrm{A}}\right) d W .
$$

The deterministic part of this system of equations describes a damped harmonic oscillation for $e_{x}, e_{p}$ where the damping and oscillation rates depend on the secondorder moments of Bob's conditioned state. The damping in these equations is not present in an analogous equation for $e_{x}$ given by Mabuchi [37] for a free particle. The equation adopted in 37 is obtained from the continuous limit of the repeated position measurement model of Caves and Milburn [11 and does not contain a noise term in the stochastic differential equation for $x$. In fact the omission of this term is in error and if the continuous limit of the repeated position measurement model of Caves and Milburn is taken correctly then the noisy contribution to $d e_{x}$, which we obtained from the SME (2.1), is in fact present. It is the damping which results from this term which leads to all observers agreeing about the conditioned state after a sufficiently long observation time. Note that after the time $\tau_{\text {col }}$ Eqs (4.2) are in fact a system of ordinary differential equations since at that point the covariances of the two conditioned states are equal. The differences in the means then damp to the steady state values $e_{x}=e_{p}=0$ indicating that Alice and Bob do eventually agree about the state of the system regardless of their initial states. Thus we have shown that the conditioned state eventually depends only on the measurement record but not the time-scale over which this occurs.

It is straightforward to use the Itô chain rule 26] to find differential equations for the expectation values of the covariance matrix for the difference between the conditioned means of Alice and Bob

$$
\begin{aligned}
\frac{1}{\omega} \frac{d}{d t}\left(E\left[e_{x}^{2}\right]\right)= & 2 E\left[e_{x} e_{p}\right]-\frac{4}{r} V_{x x}^{\mathrm{B}} E\left[e_{x}^{2}\right] \\
& +\frac{2}{r}\left(V_{x x}^{\mathrm{B}}-V_{x x}^{\mathrm{A}}\right)^{2}, \\
\frac{1}{\omega} \frac{d}{d t}\left(E\left[e_{p}^{2}\right]\right)= & -2 E\left[e_{x} e_{p}\right]-\frac{4}{r} V_{x p}^{\mathrm{B}} E\left[e_{x} e_{p}\right] \\
& +\frac{2}{r}\left(V_{x p}^{\mathrm{B}}-V_{x p}^{\mathrm{A}}\right)^{2}, \\
\frac{1}{\omega} \frac{d}{d t}\left(E\left[e_{x} e_{p}\right]\right)= & E\left[e_{p}^{2}\right]-E\left[e_{x}^{2}\right] \\
& -\frac{2}{r} V_{x p}^{\mathrm{B}} E\left[e_{x}^{2}\right]-\frac{2}{r} V_{x x}^{\mathrm{B}} E\left[e_{x} e_{p}\right] \\
& +\frac{2}{r}\left(V_{x x}^{\mathrm{B}}-V_{x x}^{\mathrm{A}}\right)\left(V_{x p}^{\mathrm{B}}-V_{x p}^{\mathrm{A}}\right) .
\end{aligned}
$$

Although we have taken the expectation value for this system of equations, the noise terms all become zero after $\tau_{\text {col }}$ so these ordinary differential equations eventually describe the whole dynamics. We are now interested in the time-scale over which the determinant of this covariance matrix becomes zero. Unfortunately the time dependence of the conditioned state variances prevents a closed form solution of this system of equations and we have not found a matrix Riccati form for the overall system. It is however straightforward to investigate the problem numerically.

We wish to show that all observers will agree about the conditioned state of the system in roughly $\tau_{\text {col }}$. To do this it is sufficent to show that an arbitary observer will agree with some preferred observer in that time. For this reason we will assume that Alice has sufficient information to describe the state of the system as a pure state and that she has access to sufficient earlier measurement records that $V_{i j}^{\mathrm{A}}(t)=V_{i j}^{\mathrm{ss}}$. An experimenter is going to be in Bob's position of not having precise knowledge of or control over the preparation of the state. We would expect that if Bob makes an accurate assessment of his initial knowledge of the state then $E\left[e_{x}^{2}\right]$ is at first of the same order as $V_{x x}$ or smaller. Since $V_{x x}^{\mathrm{B}}-V_{x x}^{\mathrm{A}}$ may be large initially, the stochastic term in the equations (4.2) for Bob's errors dominates for very short times and essentially determines a random initial condition for $e_{x}$ such that $E\left[e_{x}^{2}\right] \lesssim V_{x x}$. This reflects the fact that for short times the measurement record is dominated by noise. From this point on we find numerically that $E\left[e_{x}^{2}\right] \simeq V_{x x}$ and that all the elements of the covariance matrix damp to zero within $\tau_{\text {col }}$. Thus we have found numerically that the conditioned state depends only on the measurement record after a time equal to the time over which the conditioned state becomes pure. Most importantly we found this to be the case even where all the variances were set to very large initial values, the largest we tried being $V_{x x}^{\mathrm{B}}=V_{p p}^{\mathrm{B}}=E\left[e_{x}^{2}\right](0)=E\left[e_{p}^{2}\right](0)=10^{10}$. Thus as we anticipated, Bob can make an accurate estimate of the state within the collapse time even in the absence of accurate information about the initial state. In this case Bob's conditioned state corresponds to the maximum likelihood estimate discussed above. Interestingly, even with the pessimistic initial condition where $E\left[e_{x}^{2}\right](0)$ is significantly larger than $V_{x x}^{\mathrm{B}}(0)$ - this corresponds to Bob overestimating the accuracy of his estimate of the particle's position and the difference between his estimate and the actual value being very large - the determinant of the covariance matrix damps on the characteristic timescale $\tau_{\text {coll }}$. This feature is more pronounced for larger values of $V_{x x}^{\mathrm{B}}(0)$. Again this is because for large initial values of $V_{i i}^{\mathrm{B}}$, Bob's estimate of the system state is essentially dependent on the measurement current alone. These behaviours are demonstrated for the parameters used in the previous section in Figure (2).

From this we can conclude that after the time $\tau_{\mathrm{col}}$, any experimenter knows the state of the system regardless of how the system is initially prepared and of how much control the experimenter has over this process. In the next section when we introduce detection efficiency and thermal couplings, these will just modify the second-order moments of the conditioned state such that the steady conditioned state is no longer pure. Since the equations for the first order moments will be unmodified all that is necessary to reproduce the results of this section in this more general case is to use the collapse time $\tau_{\text {col }}$ which is 
appropriate for the new system. Although this will mean that the experimenter is left with broader position and momentum probability distributions, it will not mean that different experimenters disagree about the means of these distributions. So regardless of the detection efficiency the conditioned state is eventually uniquely determined by the measurement record and can be regarded as known by the experimenter.

\section{THERMAL AND DETECTION EFFICIENCY EFFECTS}

In order to discuss the effects of detection efficiency and other uncontrolled coupling to a bath in the model we will add an extra momentum diffusion term to the SME to obtain for $\phi=0$

$$
\begin{aligned}
d \rho_{\mathrm{c}}= & -i\left[H_{\mathrm{sys}}, \rho_{\mathrm{c}}\right] d t+2 \alpha \mathcal{D}[x] \rho_{\mathrm{c}} d t+2 \beta \mathcal{D}[x] \rho_{\mathrm{c}} d t \\
& +\sqrt{2 \alpha} \mathcal{H}[x] \rho_{\mathrm{c}} d W .
\end{aligned}
$$

This simple modification to the master equation is intended to model several possible imperfections in a real experiment. One contribution to $\beta$ is the effect of detection efficiency [6] so that $\beta$ is at least $\alpha(1-\eta) / \eta$ where the overall detection efficiency is $\eta$. The effect of cavity loss through the unmonitored mirror in a cavity QED experiment is an effective detection inefficiency. Where the loss rates out of the two mirrors are $\kappa_{1}, \kappa_{2}$, and if only the light passing through the second mirror is detected, then we get $\beta>\alpha \kappa_{1} / \kappa$ where $\kappa=\kappa_{1}+\kappa_{2}$. The best situation is if the mirror in front of the detection apparatus has significantly higher transmission than the mirror used to drive the cavity. Scattering losses of the mirror will also be an effective detection inefficiency but these are typically much smaller than transmission losses. In experiments with single atoms there will also be heating due to spontaneous emission into free space which will lead to a contribution to $\beta$ equal to $g_{0}^{2} n \Gamma / 4 \Delta^{2}$ where $\Gamma$ is the free space decay rate of the excited state of the atomic transition. Other than the restriction to the Lamb-Dicke regime this is the largest correction to the adiabatically eliminated master equation given above when a moderate detuning from the atomic transition is employed as in [1]. The spontaneous emission contribution to the heating is also proportional to the measurement coupling $\alpha$ such that $\beta_{s}=\alpha \Gamma \kappa / 4 g_{0}^{2}$ and to minimize the effect of spontaneous emission we must use cavities with the largest possible value of $g_{0}^{2} / \kappa$. Recall that if this rate is large the signal to noise of the position measurement also improves. Contributions to $\beta$ in other systems, for example the interferometric detection of the position of a moving mirror 13], will also come from any coupling of the oscillator to a thermal bath. We found that the standard quantum Brownian motion master equation [27] led to steady conditioned states that did not obey the Heisenberg uncertainty principle for small values of $r$. This a result of the non-Lindblad terms in this master equation.
The master equation we adopt here solves this problem by considering only coupling to very high temperature thermal baths for which the thermal contribution to $\beta$ is $\frac{\gamma k_{B} T}{\hbar^{2}}$, where $\gamma$ is the coupling rate to the thermal reservoir and $T$ is the temperature of the bath. This should be an adequate description of heating in the experiment as long as the bath to which the system is coupled is of sufficiently high temperature that only the diffusive evolution is significant for the timescales of interest.

There is now no SSE equivalent to this SME (5.1) and pure states become mixed during the evolution. However the SME continues to preserve Gaussian states so the previous calculation for the evolution of state can be straightforwardly modified. The second-order moments of the conditioned state still reach a steady state and we can easily find an expression for the steady conditioned state phase space area $A$ is given by

$$
\begin{aligned}
A^{\mathrm{ss}} & =\sqrt{1+\beta / \alpha}=q \geq 1 / \sqrt{\eta}, \\
s\left(\rho_{\mathrm{c}}^{\mathrm{ss}}\right) & =1-1 / q \geq 1-\sqrt{\eta}, \\
S\left(\rho_{\mathrm{c}}^{\mathrm{ss}}\right) & =\frac{q+1}{2} \ln (q+1)-\frac{q-1}{2} \ln (q-1)-\ln 2 .
\end{aligned}
$$

The linear and von Neumann entropies of the steady conditioned states are plotted for a range of detection efficiencies in Fig. (3). Even though we have effectively coupled the oscillator to an infinite temperature bath the conditioned steady states have in some sense a finite temperature but stochastically varying mean values of position and momentum.

We will consider some limiting cases here for the steady state variances in this more general situation. Where the measurement is strong $(r \ll 1)$ the position variance is insensitive to these imperfections, $V_{x x}^{\mathrm{ss}} \simeq \sqrt{r} \sqrt{q}, V_{x p}^{\mathrm{ss}} \simeq$ $q, V_{p p}^{\mathrm{ss}} \simeq 2 \sqrt{q}^{3} / \sqrt{r}$. If the dynamics dominate $(r \gg 1)$ then the position and momentum variances have the same dependence on $\beta$ as $A, V_{x x}^{\mathrm{ss}} \simeq q, V_{x p}^{\mathrm{ss}} \simeq q^{2} / r, V_{p p}^{\mathrm{ss}} \simeq$ $q$.

Finally the whole time evolution of the second-order moments of the conditioned state can be determined by solving the matrix Riccati equation. Again we will just consider the position variance as a function of time where

$$
\begin{aligned}
& V_{x x}(0)=V_{p p}(0)=V_{0}>1, V_{x p}(0)=0 \\
& \begin{aligned}
q_{x x}(t)= & \frac{+q\left(V_{0}^{2}+q^{2}\right)(c \sinh 2 b t-b \sin 2 c t)}{q^{2}\left(b^{2}+c^{2}\right)+q V_{0}\left(c^{3} \sinh 2 b t+b^{3} \sin 2 c t\right)}, \\
& +\left(V_{0}^{2}+q^{2}\right)\left(c^{2} \sinh ^{2} b t-b^{2} \sin ^{2} c t\right)
\end{aligned} \\
& b=\frac{1}{\sqrt{2}} \sqrt{\sqrt{1+\frac{4 q^{2}}{r^{2}}}-1}, \\
& c=\frac{1}{\sqrt{2}} \sqrt{\sqrt{1+\frac{4 q^{2}}{r^{2}}}+1}=q / V_{x x}^{\text {ss }}
\end{aligned}
$$

and we have scaled time by the harmonic oscillation frequency $\omega$. As before when $2 b t \gg 1$ the system reaches its steady state value and the non-linearity of the terms 
describing the conditioning of the system state mean that the time for this to occur is independent of the initial state. Interestingly this time is in fact shorter than was required to purify the conditioned state in the case of ideal detection. This is essentially because the extra noise means that past observations become irrelevant more quickly, not leaving enough time to determine a pure state completely. While the steady state is reached increasingly fast it corresponds to an increasingly high effective temperature. When $r>1$ this time to reach the steady state is $\tau_{\mathrm{s}} \simeq 2 r / q \omega=m \omega / q \hbar \alpha=\tau_{\text {col }} / q$ since in this regime $b \simeq q / r$. As was noted in the previous section the time for the conditioned state variances to reach their steady state is also the time that is necessary for different observers to agree about the system state. The time evolution of some of these quantities is plotted in Figure (4).

\section{CONCLUSIONS}

In this paper we have established that for a simple class of systems quantum trajectory theories allow the determination of a unique post-measurement state that depends only on the measurement results over a finite time. We have discussed the effects of experimental imperfections on this state determination and the analogy to classical state observation. The systems to which this analysis is applicable have the property that the conditioned density matrix is at all times Gaussian and its evolution is exactly that of the posterior probability distribution for an appropriate classical state observer. While we have considered only the case of position measurement the same treatment will be applicable to these other linear systems. Clearly in more complicated systems, such as the resonant interaction of an atom with the single mode of an optical cavity, this will not always be the case and the conditioned Wigner function will sometimes be non-positive. We would however expect that the central result we have shown here, the purity of the conditioned state after sufficiently long continuous observation and the dependence of this state on the initial state only through the measurement results, will still hold for these more interesting and complicated systems. This will however require numerical simulation of the stochastic master equation for such systems. Another feature that should generalize is the interpretation of the SME as a state observer - presumably an optimal one - for the quantum system.

\section{ACKNOWLEDGEMENTS}

A. D. would like to thank Kurt Jacobs for continuing stimulating discussions on continuous measurement theory and in particular for suggesting that it would be interesting to calculate the purity of conditioned states with and without thermal couplings. It is also a pleasure to acknowledge discussions with Hideo Mabuchi. This work is supported by the Marsden Fund of the Royal Society of New Zealand.

[1] H. Mabuchi, J. Ye, and H. J. Kimble, to be published in Appl. Phys. B (unpublished).

[2] H. M. Wiseman and G. J. Milburn, Phys. Rev. Lett. 70, 548 (1993).

[3] H. M. Wiseman, Quantum Semiclass. Opt. 8, 205 (1996).

[4] K. Mølmer, Y. Castin, and J. Dalibard, J. Opt. Soc. Am. B 10, 524 (1993).

[5] H. Carmichael, An Open Systems Approach to Quantum Optics (Springer-Verlag, Berlin, 1993).

[6] H. M. Wiseman and G. J. Milburn, Phys. Rev. A 47, 642 (1993).

[7] A. Barchielli, Int. J. Theor. Phys. 32, 2221 (1993), and references therein.

[8] N. Gisin and I. C. Percival, J. Phys. A: Math. Gen. 25, 5677 (1992).

[9] G. J. Milburn, Quantum Semiclassic. Opt. 8, 269 (1996).

[10] A. N. Korotkov, quant-ph/9808026 (unpublished); K. Mølmer, J. Mod. Opt. 44, 1937 (1997); J. Ruostekoski and D. F. Walls, Phys. Rev. A 58, R50 (1998); H. Mabuchi and H. M. Wiseman, quant-ph/9807018 (unpublished); K. Jacobs, private communication (unpublished).

[11] C. M. Caves and G. J. Milburn, Phys. Rev. A 36, 5543 (1987).

[12] P. Storey, M. J. Collett, and D. F. Walls, Phys. Rev. Lett. 68, 472 (1992).

[13] G. J. Milburn, K. Jacobs, and D. F. Walls, Phys. Rev. A 50, 5256 (1994).

[14] R. Quadt, M. Collett, and D. F. Walls, Phys. Rev. Lett. 74, 351 (1995).

[15] A. C. Doherty, A. S. Parkins, S. M. Tan, and D. F. Walls, Phys. Rev. A 57, 4804 (1998).

[16] H. M. Wiseman, Ph.D. thesis, University of Queensland, 1994.

[17] L. Diosi, Phys. Lett. 129, 419 (1988).

[18] V. P. Belavkin and P. Staszewski, Phys. Lett. A 140, 359 (1989).

[19] K. Jacobs and P. L. Knight, Phys. Rev. A 57, 2301 (1998), quant-ph/9801042.

[20] B. L. Schumaker, Phys. Rep. 135, 317 (1986).

[21] M. Rigo, F. Mota-Furtado, and P. F. O'Mahoney, J. Phys. A 30, 7557 (1997).

[22] B. M. Garraway and P. L. Knight, Phys. Rev. A 50, 2548 (1994).

[23] J. Halliwell and A. Zoupas, Phys. Rev. D 52, 7294 (1995).

[24] A. Peres, Quantum Theory: Concepts and Methods (Kluwer, Dordrecht, 1993).

[25] J. K. Breslin and G. J. Milburn, Phys. Rev. A 55, 1430 (1997).

[26] C. W. Gardiner, Handbook of Stochastic Methods for 
Physics, Chemistry and the Natural Sciences, 2nd ed. (Springer-Verlag, Berlin, 1985).

[27] C. W. Gardiner, Quantum Noise (Springer-Verlag, Berlin, 1991).

[28] W. T. Reid, Riccati Differential Equations (Academic Press, New York, 1972).

[29] H. M. Wiseman and G. J. Milburn, Phys. Rev. A 47, 1652 (1993).

[30] C. M. Caves and C. A. Fuchs, in Sixty Years of EPR, edited by A. Mann and M. Revzen (Ann. Phys. Soc., Israel, 1996), also quant-ph/9601025.

[31] W. Zurek, S. Habib, and J. P. Paz, Phys. Rev. Lett. 70, 1187 (1993).

[32] H. Mabuchi, Q. A. Turchette, M. S. Chapman, and H. J. Kimble, Opt. Lett. 21, 1393 (1996).

[33] C. J. Hood, M. S. Chapman, T. W. Lynn, and H. J. Kimble, Phys. Rev. Lett. 80, 4157 (1998).

[34] G. M. Meyer, H.-J. Briegel, and H. Walther, Europhys. Lett. 37, 317 (1997); Q. A. Turchette, Ph.D. thesis, California Institute of Technology, 1997.

[35] W. H. Zurek, Phys. Rev. D 24, 1516 (1981).

[36] D. L. Haycock, S. E. Harman, G. Klose, and P. S. Jessen, Phys. Rev. A 55, R3991 (1997).

[37] H. Mabuchi, Phys. Rev. A 58, 123 (1998), also quant-ph/9801039.

[38] J. I. Cirac, Phys. Rev. A 46, 4354 (1992).

[39] P. Whittle, Optimal Control: Basics and Beyond (Wiley, Chichester, 1996).

[40] H. M. Wiseman, Found. Phys. 28, 1619 (1998); C. A. Fuchs and A. Peres, Phys. Rev. A 53, 2038 (1996).

FIG. 1. The time evolution of (a) the unitless second-order moments $V_{x x}, V_{x p}, V_{p p}$ and (b) the two entropies of the conditioned state of the harmonic oscillator under continuous position measurement as described in the text. Note the different time axes for the entropies. Time is measured in units of the harmonic oscillator angular frequency while $r=20$. The initial state is $V_{x x}=V_{p p}=20, V_{x p}=0$ corresponding to a thermal state of the oscillator.

FIG. 2. The time evolution of Bob's mean-squared error in position $E\left[e_{x}^{2}\right]$, Bob's conditioned state variance $V_{x x}^{\mathrm{B}}$ is also plotted for comparison. In (a) the initial values are $E\left[e_{x}^{2}\right]=E\left[e_{p}^{2}\right]=5, E\left[e_{x} e_{p}\right]=0, V_{x x}^{\mathrm{B}}=V_{p p}^{\mathrm{B}}=200, V_{x p}^{\mathrm{B}}=0$ and as discussed in the text the mean squared error, although originally small, rapidly becomes of the same order as the conditioned state variance. In (b) the initial values are $E\left[e_{x}^{2}\right]=E\left[e_{p}^{2}\right]=10^{10}, E\left[e_{x} e_{p}\right]=0, V_{x x}^{\mathrm{B}}=V_{p p}^{\mathrm{B}}=10^{10}, V_{x p}^{\mathrm{B}}=0$ and over a time roughly equal to $\tau_{\text {col }}$ the mean squared error approaches zero and the conditioned position variance approaches its steady state. Note the different time-scales in the two graphs.

FIG. 3. The linear and von Neumann entropies of the steady conditioned state are plotted against effective detection efficiency. Extraneous heating of the oscillator is also described by $\eta$ as discussed in the text.
FIG. 4. (a) The time evolution of the unitless second-order moments $V_{x x}, V_{x p}, V_{p p}$ under continuous imperfect position measurement as described in the text. Time is measured in units of the harmonic oscillator angular frequency while $r=20$ and $q=5$. The initial state is $V_{x x}=V_{p p}=20, V_{x p}=0$ corresponding to a thermal state of the oscillator and for comparison the evolution of the conditioned position variance is plotted for the case of perfect detection. As discussed in the text the conditioned second-order moments reach steady state more rapidly when the detection is imperfect. (b) The mean-square error in Bob's estimate of the conditioned state mean position and Bob's conditioned state position variance for imperfect continuous position measurement $r=20, q=5$. The initial values are $E\left[e_{x}^{2}\right]=E\left[e_{p}^{2}\right]=10^{10}, E\left[e_{x} e_{p}\right]=0, V_{x x}^{\mathrm{B}}=V_{p p}^{\mathrm{B}}=10^{10}, V_{x p}^{\mathrm{B}}=0$. Even where the observation is imperfect the conditioned state is eventually independent of the initial state of the system. 

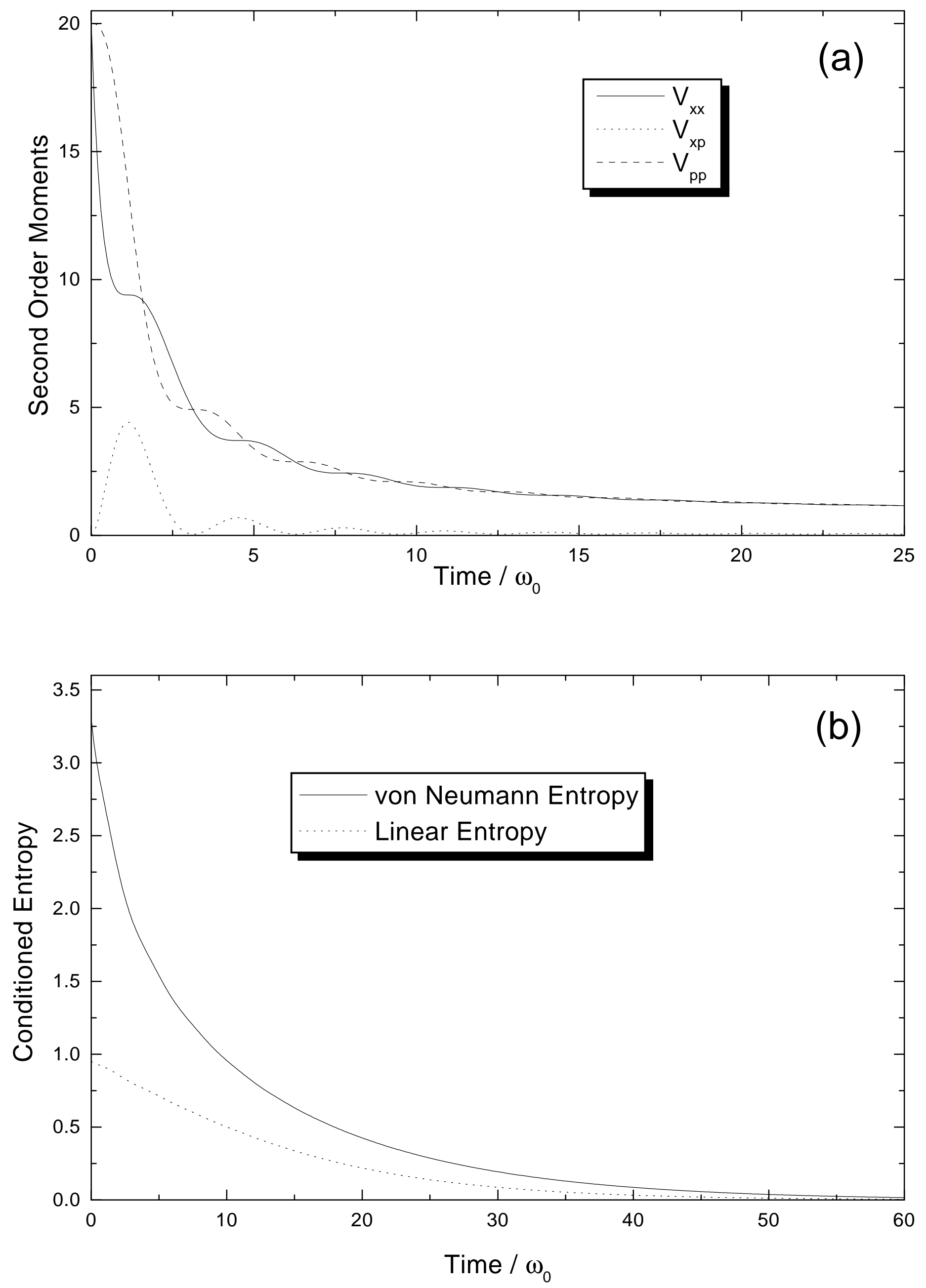

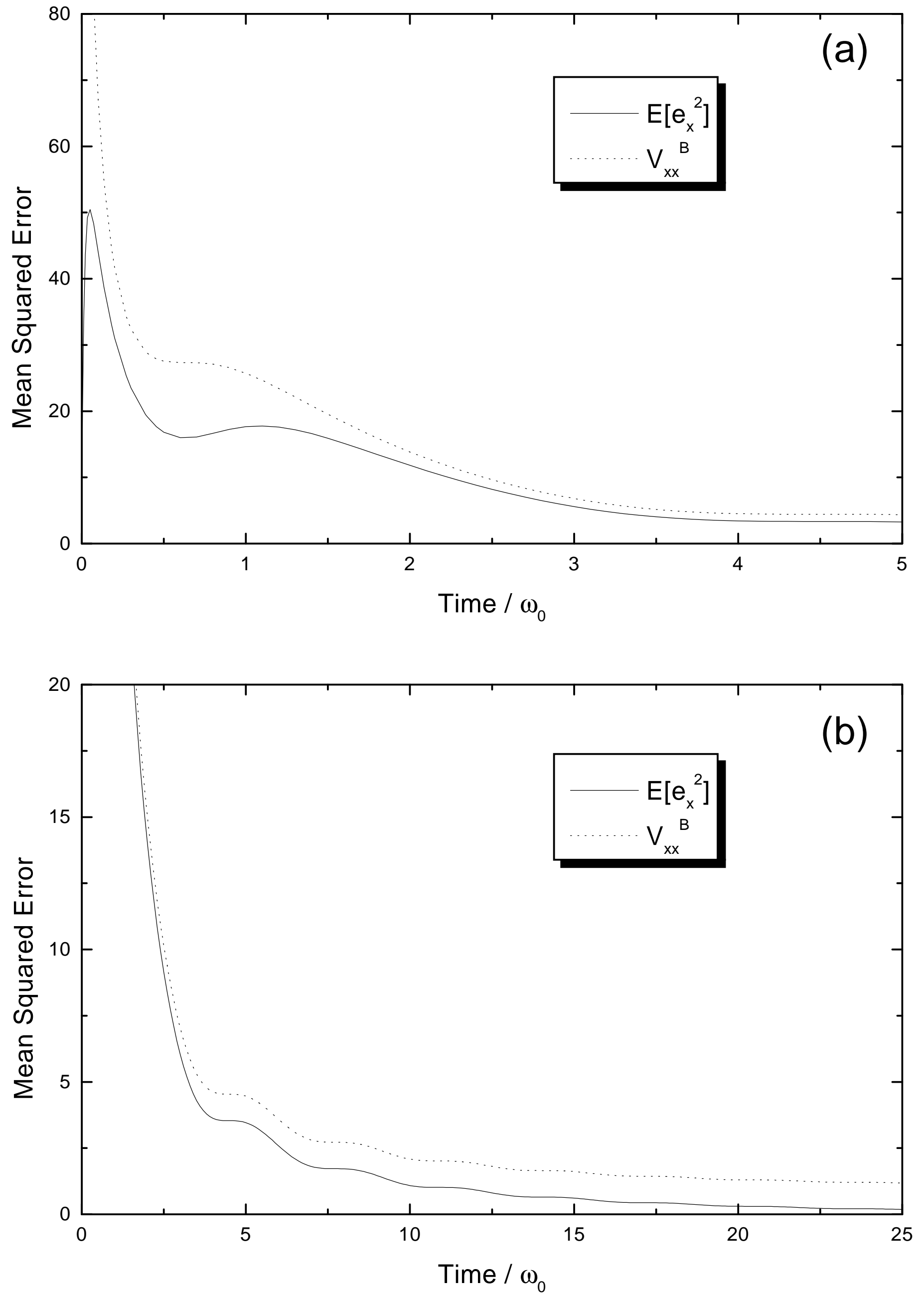


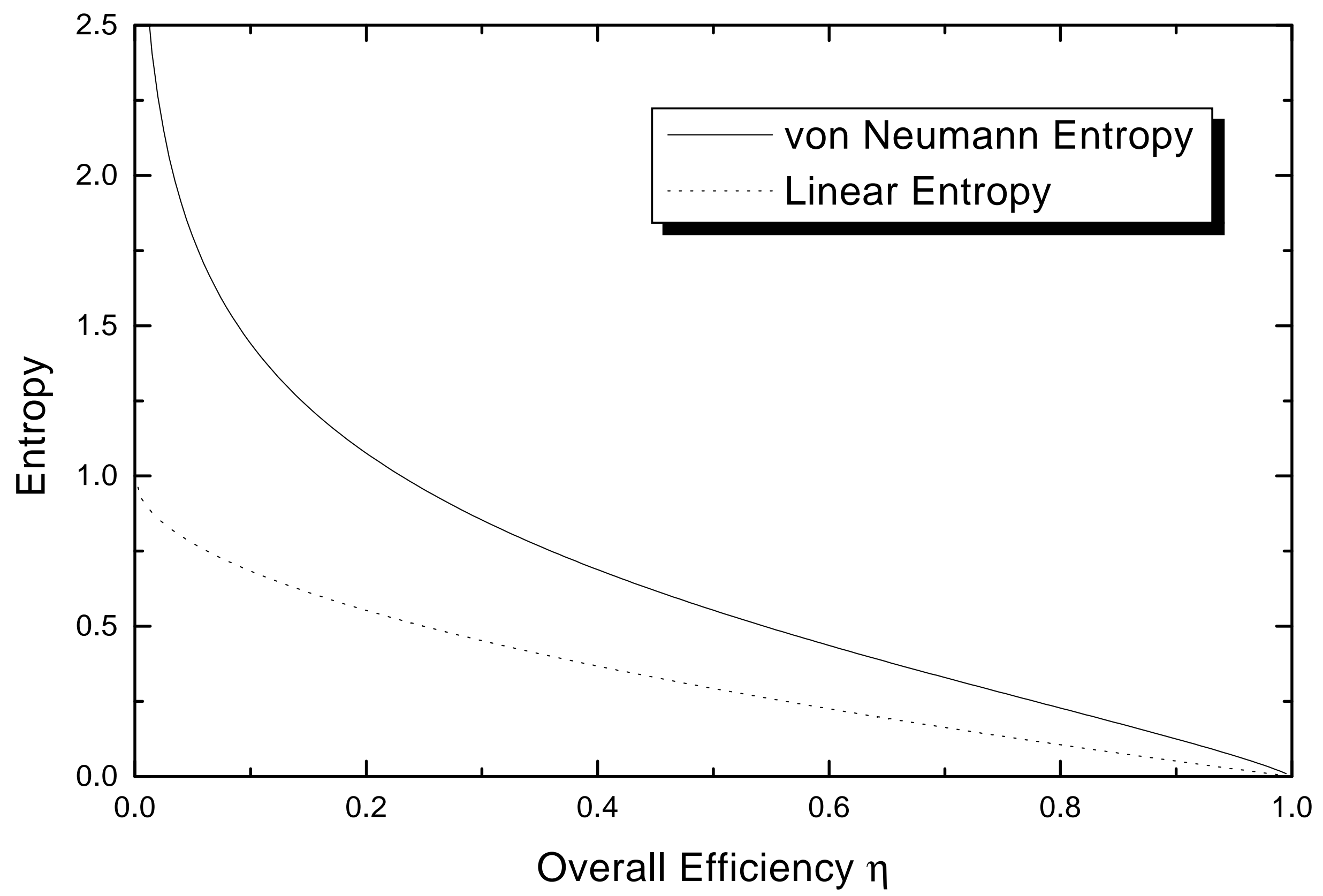



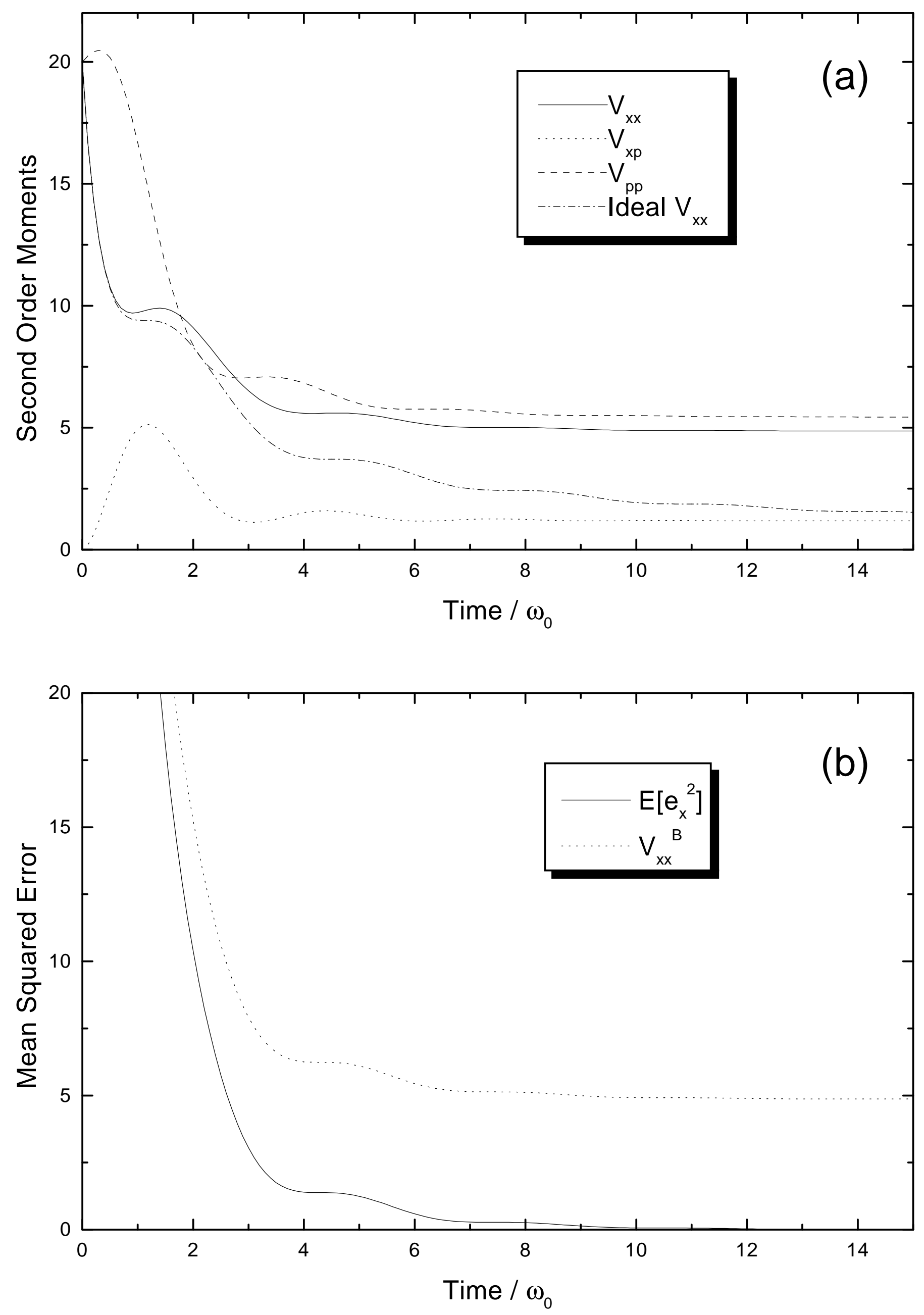